\title{
Les médecins redoutent encore plus de bureaucratie
}

\author{
Beatrix Meyera, \\ Lukas Golder ${ }^{b}$, \\ Claude Longchamp ${ }^{c}$ \\ a Cheffe du Service tarifaire \\ de la FMH et du domaine \\ SwissDRG \\ b Chef de projet senior, gfs. \\ bern \\ c Responsable de l'Institut gfs. \\ bern
}

1 Les transferts de coûts et de prestations entre le secteur hospitalier aigu et le secteur ambulatoire (hôpitaux et cabinets compris) font l'objet d'une étude séparée commandée par la FMH au Prof. S. Felder de l'Université de Bâle et au bureau B,S,S.

Volkswirtschaftliche Beratung.

2 L'étude est disponible sur www.fmh.ch $\rightarrow$ TARIFS $\rightarrow$ SwissDRG $\rightarrow$ Recherche concomitante.

Correspondance:

Service tarifaire de la FMH

Beatrix Meyer

Froburgstrasse 15

CH-4600 Olten

Tél. 0313591111

Fax 0622879690

swissdrg[at]fmh.ch
Nouvelle année, nouvelle ère pour le secteur suisse de la santé: depuis le $1^{\text {er }}$ janvier 2012, les hôpitaux de soins aigus facturent leurs prestations hospitalières au moyen du système de forfaits par cas SwissDRG. Quelles conséquences ce changement aura-t-il sur le quotidien des médecins? Pour faire toute la lumière sur les conditions de travail des médecins hospitaliers avant et après l'introduction de SwissDRG et détecter à temps les éventuelles distorsions du système, la FMH a chargé l'Institut de recherche gfs.bern de réaliser une étude concomitante à ce sujet [1]. En été 2011, l'institut a mené une première enquête représentative auprès de 1193 médecins hospitaliers et 266 médecins installés, dont vous trouverez ici les principaux résultats [2].

\section{Forte identification à la profession}

L'identification des médecins à leur profession est très forte, ce qui se traduit par un engagement élevé. En effet, $95 \%$ des médecins hospitaliers et $96 \%$ des médecins en pratique ambulatoire trouvent leur travail intéressant et varié. Une nette majorité (84\%) de médecins hospitaliers se déclarent très ou plutôt satisfaits de leur activité, pourcentage encore plus élevé chez les médecins en pratique ambulatoire (92\%). Et ce, malgré une pression élevée puisque $80 \%$ des médecins hospitaliers (fig. 1) et 79\% des médecins en pratique ambulatoire déclarent subir continuellement lors de décisions thérapeutiques. En revanche, un quart d'entre eux considèrent que leur marge de manœuvre est très faible ou faible, avis partagé par seulement $16 \%$ des médecins installés. L'influence des caisses-maladie sur le traitement est quant à elle jugée très élevée ou élevée par 16\% des médecins hospitaliers, tout comme l'influence de l'administration, également jugée très élevée ou élevée par 10\% d'entre eux. L'influence sur le choix du traitement est principalement exercée par les patients, les médecins-cadres et le personnel soignant, mais les médecins hospitaliers redoutent de voir leur liberté thérapeutique diminuer sous le système SwissDRG. Bien que les facteurs non médicaux, comme la gestion économique des ressources, soient parfaitement reconnus et pris en compte, ils ne doivent pas, en cas de doute, primer sur la décision thérapeutique ou sur le bien du patient.

\section{Eviter les doublons}

Par ailleurs, les médecins redoutent une augmentation des tâches administratives. Une nette majorité des médecins interrogés déclarent que leur charge administrative a augmenté, quel que soit le système tarifaire en vigueur dans leur hôpital. Les médecinsassistants sont les premiers concernés puisqu'ils passent déjà aujourd'hui presque autant de temps à documenter leurs prestations qu'à s'occuper des pa-

\section{En instaurant des processus efficaces, les hôpitaux pourront éviter les doublons et limiter la charge administrative.}

des pressions liées au facteur temps et aux exigences de prestations. Cependant, la forte identification et la satisfaction des médecins risquent d'être compromises par des conditions-cadres en plein changement, phénomène que les médecins pourraient attribuer à Swiss DRG. A cet égard, l'introduction de SwissDRG est notamment associée à une diminution de la liberté thérapeutique et à une augmentation de la bureaucratie.

\section{La liberté thérapeutique est essentielle}

A l'heure actuelle, 70\% des médecins hospitaliers estiment bénéficier d'une grande marge de manœuvre tients. Un tiers des médecins interrogés se plaignent d'une mauvaise organisation générale du travail dans leur hôpital (cf. figure ci-dessus) et seul un quart des médecins hospitaliers estiment pouvoir se concentrer davantage sur des tâches médicales grâce à des processus efficaces. Les médecins craignent donc que l'introduction de SwissDRG ne les éloigne encore plus des patients; c'est pourquoi il convient d'organiser les processus et l'infrastructure informatique des hôpitaux de façon à éviter les doublons lors de la documentation des prestations, et ainsi limiter la charge administrative. 
Figure 1

Les médecins hospitaliers à propos de leurs conditions de travail.

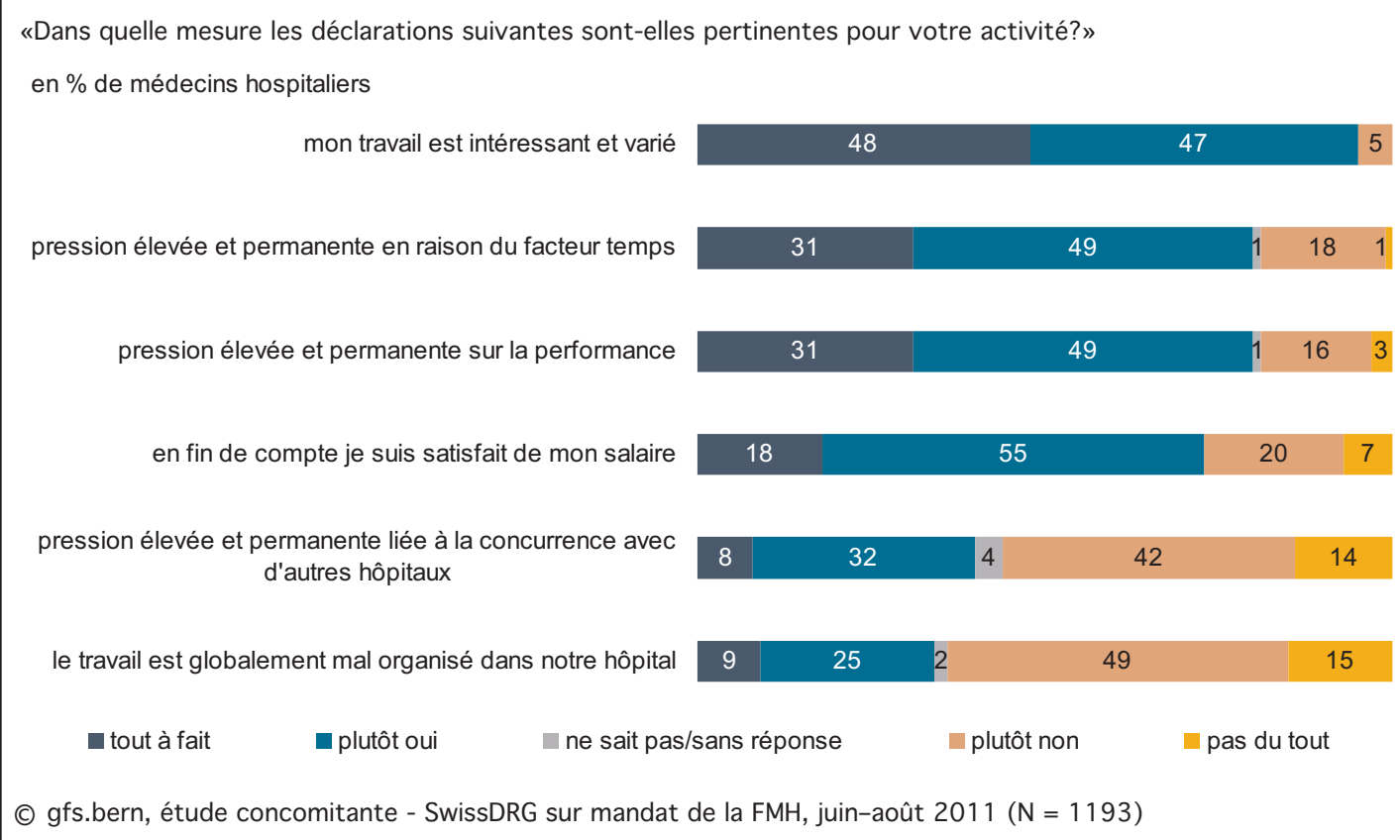

\section{Scepticisme des médecins envers SwissDRG}

La majorité des médecins (médecins hospitaliers: $60 \%$, médecins installés: $63 \%$ ) se montrent critiques face à l'introduction de SwissDRG. En revanche, les médecins hospitaliers qui travaillent depuis longtemps avec un système de forfaits par cas sont moins sceptiques: seuls $52 \%$ se montrent critiques envers SwissDRG. Mais leur évaluation repose souvent sur des connaissances limitées. En effet, seuls 21\% des médecins hospitaliers déclarent connaître très bien ou plutôt bien le système SwissDRG, 58\% disent avoir des connaissances de base et $20 \%$ affirment n'avoir aucune connaissance. Quelque $28 \%$ d'entre eux ne savent pas si leur hôpital a élaboré une stratégie en vue de l'introduction de SwissDRG; 63\% indiquent que leur hôpital dispose d'une stratégie, qu'ils jugent plutôt bonne, mais 9\% déclarent que leur hôpital n'a pas prévu de stratégie et qu'il est plutôt mal préparé aux DRG.

Ce scepticisme est une réalité, même si les médecins interrogés déclarent que les conséquences négatives comme les réhospitalisations (par ex. suite à des «bloody exits») sont dans l'ensemble peu fréquentes et même moins nombreuses dans les systèmes de forfaits par cas que dans les systèmes de forfaits par service/processus ou de forfaits journaliers. S'il est rare que les traitements des patients polymorbides soient répartis sur plusieurs séjours à l'hôpital, cela est cependant plus fréquent dans les systèmes de forfaits par cas que dans les autres systèmes.

\section{Améliorer les connaissances}

Constatant que les médecins sont toujours plus confrontés à des questions d'ordre économique, la
FMH les aide à y voir plus clair en mettant sur pied des présentations à l'intention des hôpitaux, des associations faîtières et des sociétés de discipline ainsi que des séminaires de formation continue, et en publiant des articles à ce sujet dans les médias internes et externes. Par ailleurs, elle est en train d'examiner, avec l'aide des associations faîtières, des sociétés de discipline et des hôpitaux, de nouvelles mesures susceptibles d'améliorer les connaissances des médecins en matière de facturation.

L'Institut gfs.bern réalisera une deuxième enquête dans environ une année afin de déterminer si et dans quelle mesure les craintes des médecins à l'égard de SwissDRG (augmentation de la bureaucratie, diminution de la liberté thérapeutique) se vérifient. En attendant, il s'agit de prendre ces craintes au sérieux et d'y apporter les réponses nécessaires en collaboration avec la direction des différents hôpitaux.

\section{Séminaires de formation continue}

La FMH et $\mathrm{H}+$ Formation ont agendé de nouvelles dates pour leurs séminaires de formation continue (en allemand):

- Medizin und Ökonomie: Intensivseminar für Ärztinnen und Ärzte

- SwissDRG: Seminar für Ärztinnen und Ärzte

- Ärzte und Kodierung: Verantwortung unter SwissDRG

Vous trouverez de plus amples informations sur www.fmh.ch $\rightarrow$ TARIFS $\rightarrow$ SwissDRG

Les séminaires ne sont pas sponsorisés et ne sont pas organisés dans un but lucratif. 